\title{
A RIEMANNIAN STRUCTURE FOR CORRELATION MATRICES
}

\author{
Paul DAVID AND Weiqing Gu
}

Abstract. In this paper we present a new approach to viewing the set of non-degenerate correlation matrices $\operatorname{Corr}(n)$ as a manifold and provide an optimization procedure using its newfound Riemannian structure. First we give a proof that $\operatorname{Corr}(n)$ is a quotient submanifold of the symmetric positive-definite matrices $\operatorname{SPD}(n)$ obtained via a Lie group action of positive diagonal matrices $\operatorname{Diag}_{+}(n)$. With this structure $\operatorname{Corr}(n)$ naturally inherits a Riemannian metric from $\operatorname{SPD}(n)$ and therefore enables us to develop a Riemannian-based Newton's method on $\operatorname{Corr}(n)$. We subsequently compare this Newton method to other optimization methods on $\operatorname{Corr}(n)$.

Mathematics subject classification (2010): 53-04.

Keywords and phrases: Riemannian geometry, correlation, Newton's method, quotient manifold, optimization.

\section{REFERENCES}

[1] Khaled Alyani, Marco Congedo, and Maher Moakher, Diagonality measures of Hermitian positive-definite matrices with application to the approximate joint diagonalization problem, Linear Algebra and its Applications, 528:290-320, 2017.

[2] Vincent Arsigny, Pierre Fillard, Xavier Pennec and Nicholas Ayache, Fast and simple calculus on tensors in the log-euclidean framework, Lecture Notes in Computer Science Medical Image Computing and Computer-Assisted Intervention - MICCAI 2005, page 115-122, 2005.

[3] Vincent Arsigny, Pierre Fillard, Xavier Pennec, and Nicholas Ayache, Geometric means in a novel vector space structure on symmetric positive-definite matrices, SIAM Journal on Matrix Analysis and Applications, 29(1):328-347, 2007.

[4] Z. Bai, G. Sleijpen, H. Van Der Vorst, R. Lippert, and A. Edelman, Nonlinear eigenvalue problems with orthogonality constraints, Templates for the Solution of Algebraic Eigenvalue Problems, page 281-314, 2000.

[5] Alan Edelman, Tomás A. Arias, and Steven T. Smith, The geometry of algorithms with orthogonality constraints, SIAM Journal on Matrix Analysis and Applications, 20(2):303-353, 1998.

[6] Wolfgang Förstner And Boudewijn Moonen, A metric for covariance matrices, GeodesyThe Challenge of the 3rd Millennium, page 299-309, 2003.

[7] IGOR GRUBišIĆ AND RAOUl PIETERSZ, Efficient rank reduction of correlation matrices, SSRN Electronic Journal, 2005.

[8] Mehrtash T. Harandi, Richard Hartley, Brian lovell, and Conrad Sanderson, Sparse coding on symmetric positive definite manifolds using Bregman divergences, IEEE Transactions on Neural Networks and Learning Systems, 27(6):1294-1306, 2016.

[9] Mehrtash T. Harandi, Conrad Sanderson, Richard Hartley, and Brian C. Lovell, Sparse coding and dictionary learning for symmetric positive definite matrices: A kernel approach, Computer Vision - ECCV 2012 Lecture Notes in Computer Science, page 216-229, 2012.

[10] Inbal Horev, Florian Y Ger, and Masahi SugiYama, Geometry-aware principal component analysis for symmetric positive definite matrices, 2015 ACML Conference Proceedings, 2015.

[11] Stephan Huckemann, Thomas Hotz and Axel Munk, Intrinsic shape analysis: Geodesic pca for Riemannian manifold modulo isometric Lie group actions, Statistica Sinica.

[12] Sadeep Jayasumana, Richard Hartley, Mathieu Salzmann, Hongdong Li, and MEHRTASH HARANDI, Kernel methods on the Riemannian manifold of symmetric positive definite matrices, 2013 IEEE Conference on Computer Vision and Pattern Recognition, 2013. 
[13] Takoua Kefi, Riadh Ksantini, Mohamed Bécha KaÂniche, and Adel Bouhoula, A novel incremental covariance-guided one-class support vector machine, Machine Learning and Knowledge Discovery in Databases Lecture Notes in Computer Science, page 17-32, 2016.

[14] Hyunwoo J. Kim, Nagesh Adluru, Barbara B. Bendlin, Sterling C. Johnson, Baba C. VEMURI AND VIKAS SINGH, Canonical correlation analysis on spd(n) manifolds Riemannian Computing in Computer Vision, page 69-100, 2016.

[15] J. M LeE, Introduction to Smooth Manifolds, Springer, 2012.

[16] Christophe Lenglet, MikaËl Rousson, Rachid Deriche and Olivier Faugeras, Statistics on the manifold of multivariate normal distributions: Theory and application to diffusion tensor MRI processing, Journal of Mathematical Imaging and Vision, 25(3):423-444, 2006.

[17] MAHER MOAKHER, A differential geometric approach to the geometric mean of symmetric positivedefinite matrices, SIAM Journal on Matrix Analysis and Applications, 26(3):735-747, 2005.

[18] Maher Moakher And Philipp G. Batchelor, Symmetric positive-definite matrices: From geometry to applications and visualization, Mathematics and Visualization Visualization and Processing of Tensor Fields, page 285-298, 2006.

[19] MAHER MOAKHER AND MOURAd ZÉRAÏ, The Riemannian geometry of the space of positive-definite matrices and its application to the regularization of positive-definite matrix-valued data, Journal of Mathematical Imaging and Vision, 40(2):171-187, 2010.

[20] XAVIER Pennec, Statistical computing on manifolds: From Riemannian geometry to computational anatomy, Emerging Trends in Visual Computing Lecture Notes in Computer Science, page 347-386, 2009.

[21] Xavier Pennec, Pierre Fillard, and Nicholas Ayache, A Riemannian framework for tensor computing, International Journal of Computer Vision, 66(1):41-66, 2006.

[22] Wolfgang Ring AND BENEDIKT WirTh, Optimization methods on Riemannian manifolds and their application to shape space, SIAM Journal on Optimization, 22(2):596-627, 2012.

[23] Salem Said, Lionel Bombrun, Yannick Berthoumieu, and Jonathan H. Manton, Riemannian Gaussian distributions on the space of symmetric positive definite matrices, IEEE Transactions on Information Theory, 63(4):2153-2170, 2017.

[24] Hiroyuki Sato AND Toshiniro IWAi, A new, globally convergent Riemannian conjugate gradient method, Optimization, 64(4):1011-1031, 2013. 